\title{
Letters
}

\section{Possible role of denervation-induced changes in the urothelium in the pathophysiology of cystitis in patients with spinal cord injury: a hypothesis}

Further to the excellent and contemporary article by Galloway on 'Prevention of urinary tract infecton (UTI) in patients with spinal cord injury - a microbiological review', we wish to state the following premise on the possible role of denervation-induced changes in the bladder mucosa in the development of cystitis and its sequela in patients with spinal cord injury (SCI).

\section{Presence of pathogenic microbes in the urine as the causative factor for cystitis}

Some SCI patients frequently develop cystitis without any apparent predisposing factor such as a blocked catheter, or a bladder stone. Although these SCI patients may be performing regular, atraumatic intermittent catheterisation with sterile catheters, and do not allow the bladder to get distended beyond $300 \mathrm{ml}$, they tend to develop bladder infection manifested by smelly urine, feeling unwell, urine leaks between catheterisations, and positive urine culture. These symptoms and signs disappear promptly after a course of antibiotics. So far, the emphasis had been placed on urine microbiology, and the aim was to achieve sterile urine by administering antibacterial drug therapy. Understandably, it is important to eradicate pathogenic microbes from the urine, as is indeed achieved by prescribing a course of an antibiotic to which the organism present in the urine is sensitive. This concept of achieving sterile urine with a course of antibacterial therapy is easy to implement; antibiotics are readily available on prescription; newer antibiotics are being developed; side effects of the commonly used antibacterial drug therapy are minimal; and of course, this is a well established clinical practice. However, it is difficult to maintain sterility of urine at all times in SCI patients practising intermittent catheterisation, and especially in those SCI patients with an indwelling urethral catheter or a penile sheath drainage. This raises the importance of the host tissue in the pathogenesis of urinary infection.

\section{Possible role of denervation-induced alterations in the urothelial structure and function in the increased susceptibility of SCI patients towards cystitis}

The proposed hypothesis places emphasis on the denervationinduced abnormalities in the host tissue for the development of recurrent bladder infection in patients with SCI. The structure and function of the urothelium could be altered in patients with SCI because of damage to the nerve supply to the urinary bladder. Injury to the spinal cord and consequent lack of trophic effect upon the urothelium, may lead to a cascade of events involving the urothelial proliferation, ${ }^{2}$ differentiation, maturation, and apoptosis (programmed cell death). Mucosal innervation, ${ }^{3}$ growth factors, growth factor receptors, ${ }^{4,5}$ cell adhesion molecules, secretory immunoglobulin ( $\operatorname{IgA})$, nitric oxide synthase, and cytokines may play a complex and interactive role in the development of the denervation-induced alterations in the structure and function of the urothelium; these cellular changes could eventually result in the increased vulnerability of the vesical urothelium to urinary infection.

Are there any permanent sequelae of cystitis?

Although the clinical event of cystitis usually lasts for a short period of five to ten days and produces no clinically discernible permanent damage to SCI patients, each episode of cystitis may induce certain cellular changes affecting ultimately the urothelial proliferation, maturation, and apoptosis. ${ }^{6,7}$ Such infection-induced changes, over and above the already present denervation-induced alterations in the bladder mucosa, may have a cumulative detrimental effect, thus predisposing the vesical urothelium to neoplastic transformation. An epidemiologic study of 2982 bladder carcinoma patients and 5782 population controls from ten geographic areas of the United States revealed that a history of urinary tract infection significantly elevated the risk of bladder cancer, particularly in individuals who reported three or more episodes of infection (relative risk $=2.0$ ). A history of three or more episodes of urinary tract infection was strongly related to squamous cell carcinoma in particular (relative risk $=4.8) .{ }^{8}$ Likewise, chronic infection of the urinary tract and long-term indwelling catheter increase the risk of cancer in the neuropathic bladder., ${ }^{9,10}$ Therefore, it becomes paramount to prevent cystitis in SCI patients.

\section{Clinical implications for the future}

Galloway ${ }^{1}$ critically evaluated the use of topical antiseptics applied to the perineal area and/or urethra prior to catheterisation, antiseptic solution in drainage bags, bladder washouts or instillations, oral antiseptic, acidifying and alkalinising agents, and prophylactic antibiotics in the prevention of UTI in patients with SCI. Intravesical gentamicin $(24 \mathrm{mg}$ in $100 \mathrm{ml}$ ) self-administered by intermittent catheterisation three times daily for 3 days, and weekly at home thereafter, resulted in symptomatic improvement in 10 out of 12 patients who were persistently symptomatic with positive urine cultures. ${ }^{11}$ All these methods ultimately regulate the microbial environment of the urothelium. Harris $^{12}$ in his editorial on prevention of urinary tract infections in SCI patients, made a plea for continued research preferably with multi-centre trials on new concepts, including vaccination, immunotherapy, the use of receptor analogues and bladder interference. As stated by Galloway, other approaches to prevention of UTI in patients with SCI also require to be explored besides the microbiological aspects of prevention of UTI. Thus innovative strategies need to be developed to control the denervation-induced cellular changes taking place in the urothelium so that the neuropathic bladder mucosa is no longer vulnerable to urinary infection. Knowledge of the pathophysiology of the neuropathic urothelium and cystitis ${ }^{13}$ will provide a guide towards generating future therapeutic advances aimed at (1) control of the cellular abnormalities occurring in the vesical mucosa which contribute towards its increased susceptibility 
for cystitis, and (2) minimising the consequences of its neuropathic status, especially the denervation-induced, and infection-induced proneness of the bladder urothelium to neoplastic changes. Such an approach to the modulation of urothelial function, and bacterial colonisation in SCI patients, will be in accordance with the philosophy that drugs directed against the fundamental molecular mechanisms responsible for a specific pathology are nearly always more effective and less toxic than agents that merely treat the consequences of a pathological process. ${ }^{14}$

\section{References}

1 Galloway A. Prevention of urinary tract infection in patients with spinal cord injury - a microbiological review. Spinal Cord 1997; 35: $198-204$.

2 van Velzen D et al. Vesical urothelial proliferation in spinal cord injury persons: an immunohistochemical study of PCNA and MIB. 1 labelling. Paraplegia 1995; 33: 523-529.

3 Vaidyanathan S et al. Nerve fibres in urothelium and submucosa of Neuropathic Urinary Bladder: an immunohistochemical study with S-100 and Neurofilament. Paraplegia 1996; 34: 137-151.

4 van Velzen D et al. Epidermal growth factor receptor in vesical urothelium of paraplegic and tetraplegic patients: an immunohistochemical study. Spinal Cord 1996; 34: 578.

5 Vaidyanathan S et al. p75 Nerve Growth Factor Receptor in vesical urothelium of paraplegic/tetraplegic patients: an immumohistochemical study. Submitted to Spinal Cord.

6 Elgavish A et al. Long-term treatment with lipoteichoic acid from Streptococcus faecalis affects differentiation and expression and cellular distribution of beta-1 integrins in human urothelial cells. Journal of Cellular Physiology 1996; 169: 52-65.

7 Elgavish A, Robert B, Lloyd K, Reed R. Nitric oxide mediates the action of lipoteichoic acid on the function of human urothelial cells. Journal of Cellular Physiology 1996; 169: 66-77.

8 Kantor AF et al. Urinary tract infection and risk of bladder cancer. American Journal of Epidemiology 1984; 119: 510-515.

9 Eichlorn F, Thon W, Altwein JE. The risk of cancer in neuropathic bladders. Urologia Internationalis 1984; 39: 105109.

10 El-Masri WS, Fellows G. Bladder cancer after spinal cord injury. Paraplegia 1981; 19: $265-270$.

11 George NJR, Bryden AAG. Intravesical antimicrobial therapy: efficacy without toxicity in patients with chronic bacteriuria. Journal of Urology 1997; 157: S424.

12 Harris P. Editorial: In Spinal Cord this month: Prevention of urinary tract infections in SCI patients. Spinal Cord 1997; 35: 197.

13 Callsen-Cencic P, Mense S. Differences in the up-regulation of calcitonin gene-related peptide/substance $\mathrm{P}$ vs. galanin in afferent pathways to the inflamed rat urinary bladder. Journal of Urology 1997; 157: S78.

14 Gibbs JB, Oliff A. Pharmaceutical research in molecular oncology. Cell 1994; 79: $193-198$.

S Vaidyanathan ${ }^{1}$ Ian McDicken ${ }^{2}$ BM Soni ${ }^{1}$, P Sett ${ }^{1}$

KR Krishnan ${ }^{1}$. Regional Spinal Injuries Centre. District General Hospital,

Town Lane, Southport,

Merseyside PR8 6PN,

${ }^{2}$ Department of Pathology,

The University of Liverpool,

Duncan Building,

Daulby Street,

Liverpool L69 3GA

United Kingdom.

\section{In reply to Dr S Vaidyanathan et al:}

Thank you for sending me Dr Vaidyanathan's letter on 'possible role of denervation-induced changes in the urothelium in the patho-physiology of cystitis in patients with spinal cord injury'. I wish to make these comments:

The hypothesis that Dr Vaidyanathan and colleagues propose is interesting and may help to explain why patients with a spinal cord injury (SCI) are particularly susceptible to infection even if they do not require longterm indwelling catheterisation. The long term morbidity of urinary tract infection in patients with a SCI cannot be underestimated and it is important that several approaches to the problem are addressed. The aim is to ensure adequate bladder drainage and also for the patient to comply and to be infection free if possible. In general those patients using clean intermittent catheterisation or a penile sheath have a lower incidence of urinary tract infection than those who have an indwelling catheter.

I agree that in treating urinary tract infection the causes of the infection are often ignored but they do need to be taken into consideration. If the denervation induced cellular changes can be shown to predispose to urinary tract infections, then it would be logical to look into strategies to prevent this. However it is unlikely that these strategies will ever be successful on their own and antibiotics will still play an important role in treatment, although not necessarily in the prevention of urinary tract infection.

Dr A Galloway

Consultant Microbiologist

Public Health Laboratory Service Public Health Laboratory Institue of Pathology Newcastle General Hospital Westgate Road.

Newcastle upon Tyne NE4 6BE

\section{The epiconus syndrome presenting radicular-type neurological features}

We read with interest the article by Yasumitsu Toribatake et al on 'The epiconus syndrome presenting with radicular-type neurological features' Spinal Cord (1997) 35: 163-170.

The authors show the difficulty in the diagnosis of the epiconus syndrome in their series of patients, the diagnosis was sometimes missed for years, in other instances misdiagnosis led to inappropriate surgery. They stress the importance of MRI investigations.

We believe that they failed to mention an important diagnostic tool which could aid in an earlier and more accurate diagnosis, namely electromyography (EMG). Clinical examination is also important, as is mentioned in Table 1, but an EMG is able to diagnose lower motor neuron involvement before muscle weakness and atrophy develop.

An EMG performed correctly can demonstrate involvement of one or more myotomes. If a CT-scan of the lower lumbar spine is then normal, an MRI scan of the thoracolumbar (T10-L3) spine is indicated to exclude an epiconus or proximal cauda equina lesion. This strategy greatly shortens the time interval between presentation and diagnosis. 
The EMG also gives useful additional information to help in the differential diagnosis for the other conditions mentioned in the paper.

$$
\begin{array}{r}
\text { J Willems, MD } \\
\mathrm{R} \text { Chappel, MD } \\
\text { Department of Physical Medicine and Rehabilitation } \\
\text { Middelheim Hospital } \\
\text { Lindendreef 1 } \\
2020 \text { Antwerp Belgium }
\end{array}
$$

\section{In reply to Drs Willems and Chappel}

We are grateful to Drs Willems and Chappel regarding our paper. We agree with their opinion that electromyelography (EMG) is an important diagnostic study. We think, however, that it is hard to differentiate the epiconus syndrome from lumbar radiculopathy. The main lesion of the epiconus syndrome is considered to be anterior horn cells, which are the final pathway of the motor system. Therefore, the epiconus syndrome presents almost the same abnormalities in EMG (e.g. fasciculation, fibrillation, high amplitude NMU discharge, and polyphasic discharge) as is found with lumbar radiculopathy.

It may be a good idea that a CT scan or an MRI are indicated if the EMG demonstrate one or more myotomes. However, image diagnositc studies are essential in spite of an EMG examination, because the EMG cannot provide an accurate diagnosis of the investigation of diseases, such as a disc hernia or a spinal cord tumor. Our first choice for patients with lumbar radiculopathy is an MRI of the lumbar spine. If it does not demonstrate any significant changes an MRI of the thoracolumbar spine is performed. We believe that this is non-invasive and is a time-saving strategy.

Thank you for your interest in our paper.

Yasumitsu Toribatake, MD Hisatoshi Baba, $\mathrm{MD}^{2}$ Norio Kawahara, $\mathrm{MD}^{1}$ Katsunori Mizuno, $\mathrm{MD}^{1}$

Katsuro Tomita, $\mathrm{MD}^{1}$

${ }^{1}$ Department of Orthopaedic Surgery, School of Medicine, Kanazawa University, 13-1 Takaramachi, Kanazawa 920 ${ }^{2}$ Department of Orthopaedic Surgery, Fukui Medical School, Shimoaizuki 23, Matsuoka, Fukui 910-11, Japan

\section{Multiple Thoracic Disc Herniations-Case Report and Review of the Literature}

I have recently read the article 'Mulitple thoracic disc herniations-Case report and review of the literature' by Okada et al that appeared in Spinal Cord 1997: 35: 188-186. Unfortunately I do not believe the authors had a complete review of the literature in their references.

Enclosed is a copy of our article which appeared in Spine 1992: Vol.17: S97-S102 'The natural history of thoracic disc herniation'. Some of these 55 patients had multiple disc herniations, only $15(27 \%)$ of the 55 patients eventually required surgery. Therefore, I feel strongly that the statement on page 186 is incorrect. Conservative treatment is effective in most of these cases.

I am sorry that Dr Okada et al have come to, what I feel, is a very incorrect opinion. The standard of care of most of these patients in the United States is now along the guidelines that we established in our article in 1992

\section{Dr Courtney W Brown \\ Lakewood Orthopaedic Clinic 1805 Kipling Street \\ Lakewood, Colorado 80215}

$U S A$

\section{In reply to Dr CW Brown}

I thank Dr Courtney W Brown for his comments regarding our article. He sent me a copy of his article entitled 'The natural history of thoracic disc herniation' published in Spine 1992 (Vol 17) No.6, ppS97-S102. I apologise that I could not find that article because I mainly reviewed the literature of the operative treatment for thoracic disc herniations and I was unaware of that article. Dr Courtney Brown states that surgical intervention is warranted in patients with long tract signs of myelopathy but patients without long tract signs respond well to physical therapy and activity modification. I agree with his opinion. As he states, patients without signs of myelopathy should be treated conservatively.

$$
\begin{array}{r}
\text { Dr Yoshifumi Okada } \\
\text { Department of Orthopaedic Surgery } \\
\text { Kyoto University } \\
54 \text { Kawacharacho, Shogoin, Sakyoku } \\
\text { Kyoto 606-01, Japan }
\end{array}
$$

\section{The history of modern spinal traction with particular reference to neural disorders}

I read with interest the above article by Michael V Shterenshis Spinal Cord (1997), 35, 139-146.

Dr Shterenshis said:-

'In surgery and traumatology, however, skeletal traction became a part of cervical traction procedures... When preventive measures against infection and pain complications had been secured skeletal traction became the best method of cervical traction for use over prolonged periods of time.

It was mentioned above that the main complications of halter cervical traction were pressure sores under the chin and beneath the occiput especially when applied for long periods or when used with heavy weights. Skeletal traction was free from such complications......

Nevertheless the possibility for skull tongs to penetrate the cranium and brain tissue was the main complication of the method until the beginning of the 1980s....'

However, there is another very serious complication of traction in patients with spinal cord injury that was first described by the late Terry McSweeney. Traction can lead to a deterioration of a spinally injured patient's neurological condition both by ascent of the lesion and in an increase in the depth of paralysis. I have also seen this, and Brieg instituted a systematic study of the phenoma. He wrote:

'Traumatically compressed medullary tissue is invariably attenuated and weakened. On application of skull traction, the weakened section of the compressed cervical cord is the part that is most overstretched. This inevitably results in increased neurological deficit. On scrutiny of the clinical records of 100 tetraplegic patients treated by skull traction 
at the National Spinal Injuries Centre at the Stoke Mandeville Hospital, Aylesbury, England, from 1971 to 1982, we found that therapeutic skull traction had been followed by an immediate increase in neurological deficit in $12 \%$ of the patients - a relatively high figure for the type of case in which the beneficial effects of traction were habitually anticipated. Moreover, in studies on cadavers, artificial defects in the fresh human cervical cord in situ showed typical deformation following the application of traction, confirming the basic deleterious effects of therapeutic skull traction on the injured cervical cord.'

Conversely, when the traction was released, there was considerable improvement in the neurological condition of some patients.

Thus, if skeletal traction is to be used, there must be regular monitoring of the neurological condition by regular clinical examination and serial $x$ rays to ensure that over-distraction does not take place to prevent traction upon the spinal cord.

\section{Reference}

1 Brieg A. Skull traction and Cervical Cord Injury. SpringerVerlag Berlin Heidelberg 1989.

Dr John Silver Emeritus Consultant National Spinal Injuries Centre. StokeMandeville Hospital, Bucks, England

\section{In reply to Dr $\mathbf{J}$ Silver}

I thank you for your interest in my article. There is no doubt that 'there must be regular monitoring of the neurological condition' during the skeletal traction procedure. What is more important is how to avoid this additional complication of this method of treatment.

The answer is based on the anatomy of the spine, particularly the intervertebral disc anatomy. It is well known (1) that the nucleus pulposus of the disc is a very elastic structure, and acts like a viscid fluid under applied pressure, demonstrates elastic rebound and assumes its original form upon release. It is well known also (2) that these properties can be observed in the spine of a cadaver.

It means that the intervertebral disc itself is involved with stretching of the body. The life of the disc is a constant fight against vertical forces, against compression.

By contrast, the trunk muscles, both abdominal and dorsal, being in tone, act on slight compression of the body. Trunk muscle strength counteracts the incompressible discs. In a healthy body this spine-muscles stretchingcompressing mechanism is in good balance. This balance slightly shifts to stretching during sleep at night. This is why in the morning a human being is taller than he is in the evenings.

Tetraplegic patients and cadavers have no muscle strength. This means that their spines are already overstretched!

In my opinion, not historical but neurological, one should treat tetraplegic patients more by 'immobilization' or 'fixation' than 'traction'. If however, the skeletal traction procedure is used, the force should be significantly less compared to the force applied to patients with normal muscle tone.

\section{Reference}

1 Rothman RH, Simeone FA. (eds). The Spine. 3rd ed., Philadelphia, London, Toronto. (1):p.47; (2):p.48. WB Saunders Co.: 1992.

Dr MV Shterenshis 